\title{
EXACT CALCULATIONS OF PHASE AND MEMBRANE EQUILIBRIA FOR COMPLEX FLUIDS BY MONTE CARLO SIMULATION
}

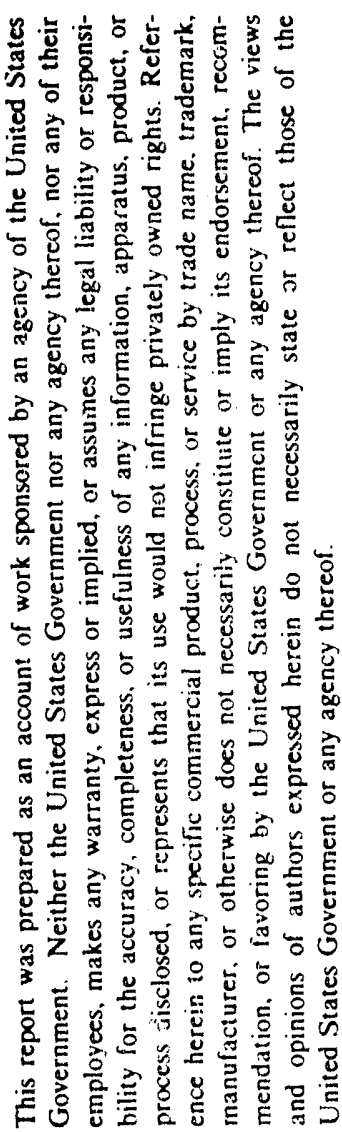

\author{
Progress Report \\ and \\ Continuation Award Application \\ U.S. Department of Energy Project \# DE-FG02-89ER14014 \\ Chemical Sciences Division, \\ Office of Energy Research
}

August 28, 1990

Principal Investigator

Athanassios Z. Panagiotopoulos

School of Chemical Engineering,

Comell University, Ithaca, NY 14853, U.S.A.

TÁBLE OF CONTENTS

1. PROGRESS TO DATE $\ldots \ldots \ldots \ldots \ldots \ldots \ldots \ldots \ldots \ldots \ldots \ldots$

1.1 Technical achievements ....................... 1

1.2 Publications and Presentations resulting from this work $\ldots \ldots \ldots \ldots$. 5

1.3 Personnel associated with the project .................. 7

2. PLAN FOR THE NEXT BUDGET PERIOD $\ldots \ldots \ldots \ldots \ldots \ldots \ldots \ldots . \ldots$

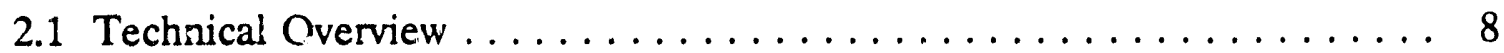

2.2 Personnel needs $\ldots \ldots \ldots \ldots \ldots \ldots \ldots \ldots \ldots \ldots \ldots \ldots \ldots, 11$

2.3 Equipment needs $\ldots \ldots \ldots \ldots \ldots \ldots \ldots \ldots \ldots \ldots \ldots \ldots \ldots \ldots$

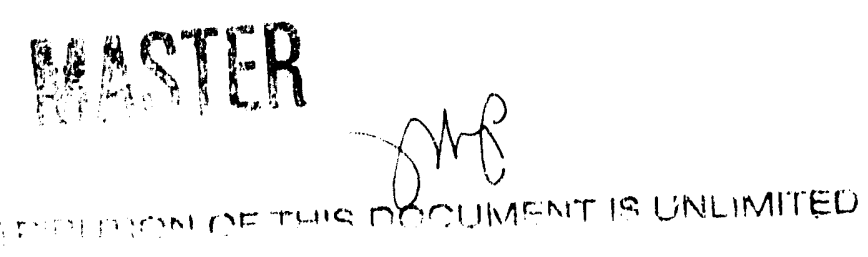




\section{PROGRESS TO DATE}

\subsection{Technical achievements}

The general objective of this project is the investigation of phase equilibria for complex fluids using a novel methodology, Monte Carlo simulation in the Gibbs ensemble. The methodology enables the direct determination of the properties of two coexisting fluid phases (e.g. a liquid at equilibrium with its vapor) from a single computer experiment, and is applicable to multicomponent systems with arbitrary equilibrium constraints imposed. The specific goals of this work are to adapt the Gibbs technique to (a) highly asymmetric mixtures with large differences in size and potential energies of interaction (b) chain molecules and (c) ionic systems. Significant progress has been made in all three areas. In the following paragraphs, we will briefly describe the progress made in each area, using the same numbering scheme for the tasks as in the original proposal.

\section{a. Task I. Highly Asymmetric Systems}

Highly asymmetric mixtures have components that differ greatly in size or strength of attractive interactions. They are common in industrial practice, and very often it is precisely the large degree of asymmetry that makes a specific separation process work (for example, supercritical fluid extraction processes). Few resulis for the chemical potential and excess thermodynamic properties of asymmetric mixtures exist ${ }^{1,2}$ and the asymmetry of model systems studied covers a fairly narro ; range of size ratios. However, it is precisely for these systems that the disagreement between exact simulation results and current theories of mixiures is most pronounced. The discrepancies in the excess thermodynamic properties grow rapidly with increasing asymmetry of the mixtures.

The original version of the Gibbs methodology for molecular simulation of phase equilibria ${ }^{3}$ is not efficient when applied to the calculation of phase equilibria of highly asymmetric mixtures, because the probability of transfer of molecules with large size can become prohibitively low. The solution to this problem was a modification to the Gibbs methodology that is based on particle identity exchange. With particle identity exchange, we can successfully perform calculations of phase diagrams for mixtures of spherically symmetric components that differ in size by a factor of at least eight.

1 K.S. Shing and K.E. Gubbins, "The chemical potential in non-ideal liquid mixtures. Computer simulation and theory", Molec. Phys. 49, 1121-1138 (1983).

2 K.P. Shukla and J.M. Haile, "Computer simulation results for thermodynamics excess properties in fluid mixtures. I. Effects of atomic size in simple mixtures", Molec. Phys., 62, 617 (1987).

3 A.Z. Panagiotopoulos, "Direct determination of phase coexistence properties of fluids by simulation in a new ensemble", Molec. Phys., 61, 813 (1987). 
Our studies focused on two distinct aspects of the behavior of highly asymmetric mixtures. The first question we addressed was the behavior of mixtures as they approach the point of liquid-liquid immiscibility. The results, described in reference A.1 (section 1.2) indicate that a reduction of the strength of unlike-pair interactions below $75 \%$ of their geometric-mean value leads to liquid-liquid demixing. The simulation results are well described by a statistical-mechanical virial equation of state ${ }^{4}$.

In a second study, we performed a systematic investigation of the phase behavior of mixtures of Lennard-Jones particles in which the unlike-pair interactions are described by conventional LorentzBerthelot combining rules. The range of parameters covered was energy ratios of up to two, and volume ratio up to eight. This range far exceeds that of previous calculations of excess thermodynamic properties for similar mixtures. Comparisons of the results with a simple theoretical model (the van der Waals 1-fluid theory of solutions) revealed surprisingly good agreement. Typical results are shown in Figure 1, taken from reference A.3 (section 1.2 of this report), in which Monte Carlo results for the phase behavior of systems with size parameter ratio of $\sigma_{22} / \sigma_{11}=1.5$ and a range of energy parameter ratios are compared with theoretical calculations. We conclude that even simple solution theories have a much wider range of applicability in terms of their ability to predict phase equilibria than previously believed. These results provide some theoretical justification to the apparent success of semi-empirical engineering equations of state.
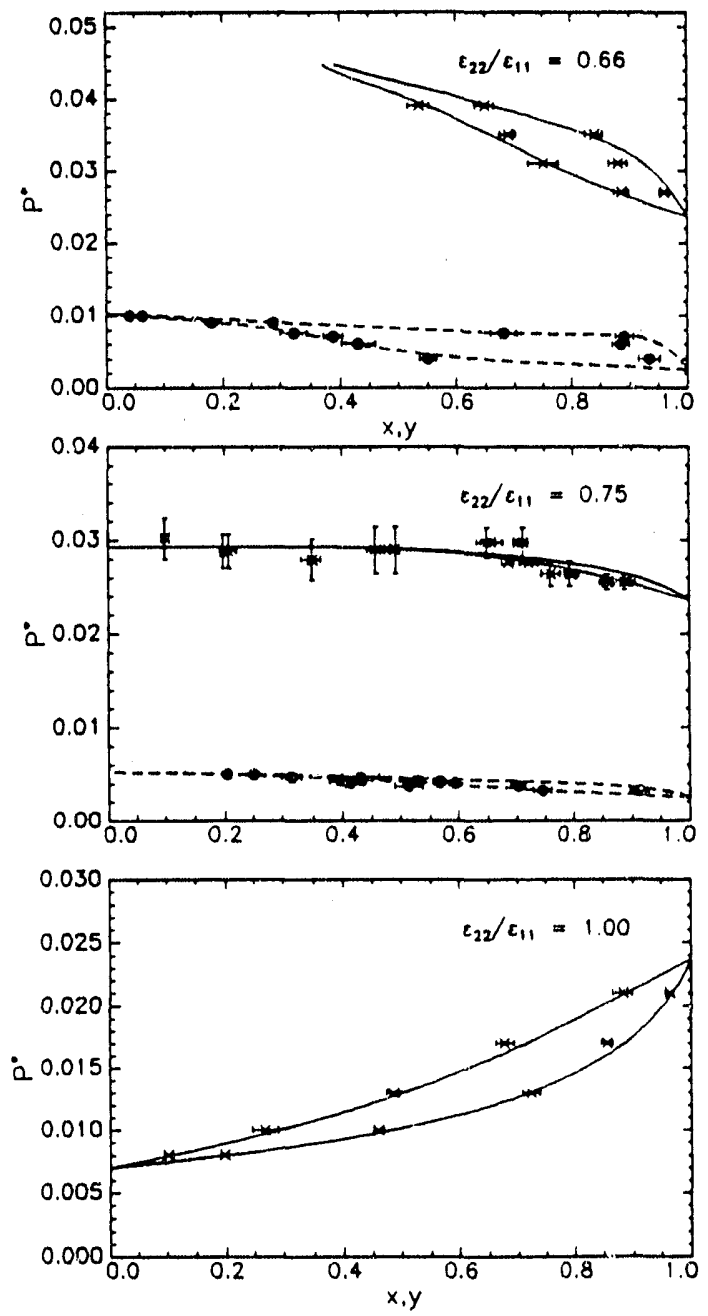

Figure 1. Results for asymmetric systems with $\sigma_{22} / \sigma_{11}=$ 1.50. Monte Carlo: $(x) T^{*}=1.00 ;(\bullet) T^{*}=0.75$. Theory: $(\longrightarrow) T^{*}=1.00 ;(--) T^{*}=0.75$

4 C.J. Peters, and R.N. Lichtenthaler, Physica A156́, 417-435 (1989). 


\section{b. Task II. Multisegment arst Chain Molecules}

Many systems of industrial importance contain molecules that are far from being spherical in shape. A useful approximation for the molecular-level description of such molecules is to consider them as composed of a number of segments, or functional groups. The available techniques for modelling the thermodynamic properties of such materials are primarily excess Gibbs energy or equation-of-state methods, or, at the limit of very long molecules, expressions that take into account the entropy-of-mixing effects for long chains (Flory-Huggins theory). No fully satisfactory theoretical or simulation-based techniques are available for molecules that belong to this class. However, it is precisely for such systems that the development of improved theoretical techniques would have the most significant practical impact. Recent simulation work on phase equilibria of lattice-based polymer/solvent systems ${ }^{6}$ covers primarily the liquid branch of the coexistence envelope away from the critical point.

Two new developments are presently pursued in our group. The first is the extension of the Gibbs methodology to lattice systems. The primary difficulty in applying the Gibbs technique to lattice systems is the need for mechanical equilibration via changes in the volume of the coexisting regions. In continuous systems, these changes can be performed gradually with only minimal excursions away from local equilibrium. For lattice systems, only changes that correspond to a full lattice layer (in three dimensions) are possible for systems with periodic boundary conditions. We are presently exploring several possible remedies of this difficulty. For example, elimination of the requirement for periodic boundary conditions may lead to only minor disturbances to the equilibrium state of the system while permitting gradual (and thus efficient) changes in density of the two regions. We expect that results from ovir simulations will significantly aid in discriminating among conflicting predictions of polyrner solution theories as to the location of coexistence curves in polymer systems ${ }^{7}$.

The second development in this area is a new methodology for the calculation of chemical potentials (and by a simple extension to a Gibbs-type methodology, of phase

5 J.M. Prausnitz, R.N. Lichtenthaler and E.G. de Azevedo, Molecular thermodynamics of fluid-phase equilibria, Prentice-Hall (1986).

6 W.G. Madden, A.I. Pesci and K.F. Freed, "Phase-equilibria of lattice polymer and solvent - Tests of theories against simulations", Macromolecules, 23, 1181-91 (1990).

7 I. Szleifer, "A new mean-field theory for dilute polymer solutions: Phase diagram, conformational behavior and interfacial properties", J. Chem. Phys., 92, 6940-52 (1990). 
diagrams) for long polymers. For small molecules, the most successful approach to the calculation of chemical potentials is via Widom's formula ${ }^{8}$ :

$$
\mu^{\mathrm{ex}}=-k_{\mathrm{B}} T \ln \left\langle\exp \left(-U_{\text {test }} / k_{\mathrm{B}} T\right)\right\rangle
$$

where $\mu^{\text {ex }}$ is the excess chemical potential of a component and $U_{\text {test }}$ is the "test particle" energy experienced by a particle randomly placed in the solution of interest. This approach fails for polymers because the low-energy configurations that contribute significantly to the ensemble average for the chemical potential are extremely rare. Grand-canonical or Gibbsensemble Monte Carlo simulations that also rely on particle insertions for imposing or equilibrating chemical potentials fail for polymers for essentially the same reason - it is exceedingly difficult to randomly place a polymer molecule in a dense solution with no overlaps with existing molecules. Our new approach is based on abandoning the point of view of the full polymer molecule, and instead concentrating on the segments of which it is composed. We thus employ Widom's equation for calculating chemical potentials of single segments belonging to a long polymeric molecule. At the limit of long chains, the chemical potential per segment is essentially constant, and the chemical potential of the full polymer can be recovered. We have already demonstrated that this technique is practical for long polymers, and are presently in the process of preparing a publication with our results (reference A.4 in section 1.2).

\section{c. Task III. Ionic Systems}

Electrolyte solutions are common in industrial practice, including the rapidly growing biotechnology industry. Common types of equilibria encountered are between a liquid and a gas phase (gas scrubbing, salting out, distillation), or two liquids (extraction of metals in hydrometallurgy). Molten salts are used in the production of metals by electrochemical methods. Commonly used methods for handling ionic equilibria include first-order approximations such as the Debye-Hückel theory modified to take into account solution nonidealities. These models are successful only at the expense of introduction of a number of adjustable parameters fitted to experimental data. The problems are especially severe for higher ionic strengths. Dense ionic systems, such as molten salts are beyond the capabilities of this approach. Theoretically based techniques, such as perturbation theories and integral equation methods, have been successful in describing the thermodynamics and structure of ionic systems for certain cases, but generally apn!icable theoretical techniques are not yet available.

A significant effort during the present budget period has been devoted to development of appropriate methodologies for the calculation of equilibria in ionic systems. Two primary problems, the handling of long-range coulumbic forces and the question of

8 B. Widom, "Some Topics in the Theory of Fluids", J. Chem. Phys., 39, 2808-12 (1963). 
electroneutrality during the transfer steps were successfully resolved. After extensive tests of many alternatives, it was concluded that it is necessary to use a full summation for an infinite periodic system of the long-range forces (Ewald sum) to achieve results that are physically reasonable and approximately independent of sirnulation system size. In previous simulations of chemical potentials for electrolyte systems ${ }^{9}$, the electroneutrality requirement imposed the need for simuitaneous insertions of pairs of ions. At low temperatures and high densities, which is the regime of interest for phase equilibrium calculations, this approach becomes impractical. It was determined that a simple temporary relaxation of the requirement for electroneutrality during attempted transfers of particles in Gibbs-ensemble simulations leads to correct results.

Results of our calculations for the restricted primitive model of electrolyte solutions (charged hard spheres of equal diameter) are presented graphically in Figure 2 (from reference A.3 in section 1.2). Our simulation results differ substantially from theoretical estimates for the phase coexistence envelope of this simple model. These results represent the first detailed calculations of phase behavior of in ionic system by simulation. It is hoped that the availability of exact results for these systems will facilitate the development of more accurate theories for handling ionic solutions.

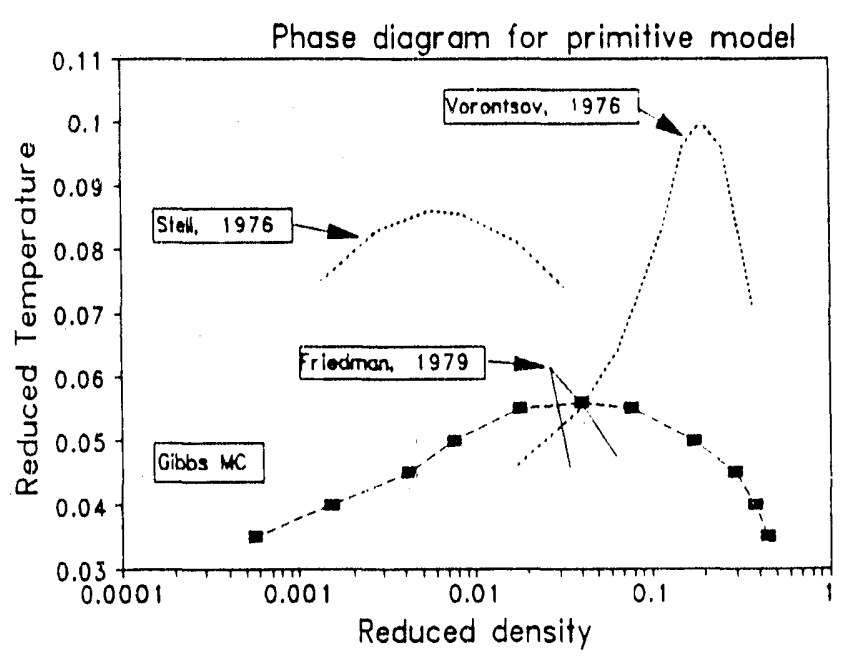

Figure 2. Phase diagram for the restricted primitive model for electrolyte solutions.

\subsection{Publications and Presentations resulting from this work}

\section{A. Refereed Publications in Preparation}

1. M.E. van Leeuwen, C.J. Peters, J. de Swaan Arons and A.Z. Panagiotopoulos, "Investigation of the transition to liquid-liquid immiscibility for Lennard-Jones systems", to be submitted to Fluid Phase Equil.

2. A.Z. Panagiotopoulos, "Phase equilibria of charged hard sphere fluids from Gibbsensemble simulations", to be submitted to J. Chem. Phys. 
3. V.I. Harismiadis, N.K. Koutras, D.P. Tassios and A.Z. Panagiotopoulos, "How good is conformal solutions theory for phase equilibrium predictions?", to be submitted to Fluid Phase Equil.

4. S.K. Kumai and A.Z. Panagiotopoulos, "A new method for molecular-based calculations of chemical potentials and phase equilbria for polymers", to be submitted to Phys. Rev. Lett.

\section{B. Oral Presentations}

1. "Molecular Simulation of Phase Equilibria" poster presentation at the annual 1989 CCR meeting in Parsippany, NJ, Oct. 1-3, 1989.

2. "Molecular Simulation of Phase Equilibria", presented at the National Center of Research in the Physical Sciences 'Demokritos', Athens, Greece, Jan. 11, 1990.

3. "Molecular Simulation of Phase Equilibria", Univ. of Houston (Chem. Eng. Dept. seminar speaker), March 16, 1990.

4. "Molecular Simulation of Phase Equilibria", Princeton Univ. (Chem. Eng. Dept. seminar speaker), May 2, 1990.

5. "Gibbs Ensemble Simulations of Fluid Phase Equilibria", plennary lecture at the Third Liblice conference on Liquids, Bechyne, Czechoslovakia, May 28-June 1, 1990.

6. "The Gibbs Method for Calculating Phase Equilibria by Simulation", seminar presented at Shell Laboratories, Amsterdam, the Netherlands, June 3, 1990.

7. "Phase equilibria of ionic fluids", seminar presented in Universtität Karisruhe, June 4, 1990.

8. "Molecular Simulation of Phase Equilibria", Univ. of California, Santa Barbara (Chem. Eng. Dept. seminar speaker), Oct. 4, 1990.

9. "Molecular Simulation of Phase Equilibria", Univ. of California, Berkeley (Chem. Eng. Dept. seminar speaker), Oct. 8, 1990.

10. "Gibbs-Ensemble Simulations of Ionic Systems", paper accepted for presentation at the Annual AIChE meeting, Chicago, Illinois, Nov. 11-16, 1990. 


\subsection{Personnel associated with the project}

During the first budget period, the following researchers have been associated with various stages of the present project.

1. Athanassios Z. Panagiotopoulos (Principal Investigator).

2. Post-doctoral Associates

Igal Szleifer (Ph.D, Technion U., 1987). Dr. Szleifer came to Cornell in 1989 as a post-doctoral associate in the Department of Chemistry (with Prof. B. Widom). Since June, 1990, he has been working on extensions of the Gibbs-ensemble methodology to lattice models of polymer solutions. Dr. Szleifer has significant prior expertise in theoretical modelling of polymeric systems.

3. Graduate Students

Wendy Molle (B.S., U. Buffalo, 1988). Ms. Molle came to Cornell as a first-year graduate student in August 1988. She has been working on ionic systems simulations since January 1989. While she had made a significant amount of progress in terms of taking courses and developing the required computational skills, she was unfortunately unable to continue with the project, due to serious health problems. She is presently on leave of absence from Cornell, and might rejoin the project at a later time.

Makis Orkoulas (B.S., U. of Patras, 1989). Mr. Orkoulas came to Cornell as a second-year graduate student in June 1990. His doctoral thesis project is simulations of phase equilibria of ionic systems. He will be fully supported by funds from the present project in the coming budget period.

Alan Mackie (B.S., U. of Edinburgh, 1989). Mr. Mackie came to Cornell as a firstyear graduate student in September 1989. He is co-supervised by Profs. D. Hammer and A. Panagiotopoulos (both in the School of Chemical Engineering). His thesis topic is molecular simulation studies of bilayers and their interactions with proteins. Mr. Mackie is presently st'dying the phase behavior of model multisegment surfactant molecules composed of a number of hydrophilic and hydrophobic residues. In the coming budget period, he will be supported at a $50 \%$ level by funds from the present project.

4. Undergraduate Students

Monica van Leeuwen

Vassilis Harismiadis

Nikos Koutras 


\section{PLAN FOR THE NEXT BUDGET PERIOD}

\subsection{Technical Overview}

Our activities in the coming budget period will continue to focus on the three main tasks outlined in the original proposal. While we have made significant progress in all areas, the first budget period was primarily one of personnel training and computer program development, with the bulk of our results expected in the second and third budget periods. Specific plans for the three main tasks are outlined in the paragraphs that follow. Anticipated personnel and equipment needs are presented in subsequent sections.

\section{a. Task I. Highly Asymmetric Systems}

This task is close to completion, with all method development and computer programming activities essentially finished. We also have results available for a number of model systems that cover a wide range of size and energy parameter ratios. The main conclusion from our work to date has been that simple solution theories have a much greater range of applicability to predictions of phase equilibria of asynunetric systems than previously thought. We have not yet established firm limits as to how different the components need to be before present solution theories start breaking down. Establishing these limits for size/energy asymmetry will be the subject of detailed study in the forthcoming budget period.

\section{b. Task II. Multisegment and Chain Molecuies}

The novel technique for calculation of chemical potentials for chain molecules that was described in section 1.1 will form the basis for calculations in the coming budget period of equilibria in model and realistic systems with chain molecules. Systems to be studied include:

(a) Long-chain polymers for which a flexible model is appropriate. A particularly attractive model is a flexible chain with excluded volume interactions and a harmonic bond potential. The reason the harmonic bond potential is preferred over a model with fixed bond lengths is that the discontinuities in the potential imposed by fixed bond lengths make it difficult to obtain mechanical quantities (such as pressure), or perform volume changes in the mechanical equilibration step of the Gibbs technique. Monte Carlo and theoretical calculations for a similar model are available ${ }^{10}$ and can be used to validate the Gibbs simulations. Previous Monte Carlo results for model systems of this type have facilitated the development of improved theoretical

$10 \mathrm{~J}$. Gao and J.H. Weiner, "Contribution of covalent bond force to pressure in polymer melts", J. Chem. Phys., 91, 3168-73 (1989). 
techniques for describing the free energy for polymeric systems ${ }^{11}$. The Gibbs simulation results should further contribute towards this goal.

(b) For closely approximating real multisegment fluids, realistic potentials that include torsional potentials for rotation around single bonds ${ }^{12}$ will be utilized.

(c) Multisegment surfactant molecules composed of a number of hydrophobic and hydrophilic residues. Studies of such systems are relevent to the development of new separation methods using organized solution microstructures (normal or reverse micelles) and are also important in understanding interactions involving cell surfaces.

An additional possibility for application of the Gibbs methodology for polymer systems that does not rely on successful transfers of the chain molecules will be explored. In many applications, one is primarily interested in equilibrium between a phase containing a polymer and a phase that only contains small components. An example of such a case is the sorption and swelling of polymers when contacted with small solutes (such as simple hydrocarbon gases). Frequently, a polymer phase that is initially in a glassy state, swells and changes into a rubbery state on sorption of small components that act as plasticizers. Several separation techniques have been proposed that rely on differences in solubility of a component in a polymer matrix. This type of problem is ideally suited for study using the Gibbs methodology. Only the small species is present in both phases, and therefore only transfers of this component need to be performed. The method would allow the calculation of the solubility and changes in the structure of a polymer on sorption from first principles, and would thus be a major advance in the understanding sorption of small solutes in polymers.

\section{c. Task III. Ionic Systems}

We have completed the investigation of the phase coexistence curve and critical point for the restricted primitive niodel (charged hard spheres of equal diameters) in the previous budget period.

Equilibria involving non-primitive models for electrolytes will be studied during the coming budget period. Potertial parameters appropriate for simulations of ionic melts of

11 M.G. Bawendi and K.F. Freed, "Systematic corrections to Flory-Huggins theory: Polymer-solvent-void systems and binary blend-void systems", I. Chem. Phys., 88, 2741-56 (1988).

12 D.N. 'Theodorou and U.W. Suter, "Detailed molecular structure of a vinyl polymer glass", Macromolecules, 18, 1467-1478 (1985). 
molten alkali halides are available ${ }^{13}$. It is easy to incorporate additional forces between the charged particles (such as soft-core repulsions and van der Waals attractions) in Gibbs simulations in order to obtain realistic models for specific electrolytes. One of the objectives of the phase equilibria calculations in this part of the work will be to determine how the critical points and phase equilibria expected for the restricted primitive model are affected by intermolecular forces present in real electrolytes that are not taken into account in the primitive model. Comparisons with experimental data for liquid-liquid phase separations of aqueous electrolyte solutions ${ }^{14}$ will be made.

An important class of systems that are not adequately described by present theoretical treatments is the thermodynamics of binary mixtures of an ionic and a non-ionic (polar or non-polar) component ${ }^{15}$. A good model system for these mixtures is the $\mathrm{NaOH} / \mathrm{H}_{2} \mathrm{O}$ system that is believed to have a continuous critical curve connecting the critical point of water to the hypothesized critical point of $\mathrm{NaOH}^{16}$. Studies of model mixtures of this type will provide for the first time a unified description of phase behavior of a continuous spectrum of systems of character intermediate between fully ionic and fully non-ionic. In addition to phase behavior, the conductance characteristics of the solution will be studied by determining the fraction of the ionic component present in associated (ionpaired) form.

An additional type of equilibria to be studied involves ionic species in the presence of semipermeable membranes (Donnan equilibria), which are of significant interest for understanding processes in living organisrns, and in the development of membrane separations for fermentation products. The requirements for accuracy of the calculations in this case are extreme, as smail pressure differences are normally associated with this type of equilibria. Of particular importance would be the study of systems with large, multivalent ions (representative of protein molecules). The presence of such species will not result in sampling problems for the transfer step of the Gibbs method, since for membrane equilibria there is no requirement for equality of the chemical potential of the species that are not membrane-permeable. Theoretical treatments of the Donnan equilibrium problem are

13 A. Baranyai, I. Ruff and R.L. McGreevy, "Monte Carlo simulation of the complete set of molten alkali halides", J. Phys. C: Solid State Phys., 19, 453-465 (1986).

${ }^{14} \mathrm{H}$. Weingärtner, "Thermodynamics of aqueous electrolyte solutions with liquid-liquid phase separation", Ber. Bunsenges. Phys. Chem., 93, 1058-65 (1989).

15 J.C. Tanger and K.S. Pitzer, "Thermodynamics of $\mathrm{NaCl}-\mathrm{H}_{2} \mathrm{O}$ : A new equation of state for the near-critical region and comparisons with other equations for adjoining regions, Geochemica Cosmochimica Acta 53, 973-87 (1989).

${ }^{16}$ U. Franck, Universität Karlsruhe, personal communication 
available ${ }^{17}$, and will be used for comparisons with the simulation results. It is hoped that the availability of exact results for these systems will greatly facilitate the further development of theoretical techniques for the description of electrolyte systems.

\subsection{Personnel needs}

It is expected that in the coming budget period, one additional full-time student will be added, in the area of phase equilibria of polymer systems. The proposed budget request for the coming year reflects this fact, and takes into account an expected rollover of funds from the present fiscal period.

\subsection{Equipment needs}

The primary need not presently adequately addressed by existing computing equipment is the ability for rapid visualization of three-dimensional configurations of particles (atoms, molecules or ions). Significant advances in workstation technical capabilities accompanied by substantial improvements in price/performance ratios have been realized in the recent months. The acquisition of a graphics workstation with 3-D visualization capabilities is expected to significantly enhance the computing environment necessary to support the present project. A typical configuration of equipment from a specific vendor is given below.

Cost

(after Cornell discount)

DECstation 5000/200PXG with 19" color monitor, 3-D graphics rendering board, 8 color planes, $16 \mathrm{Mb}$ of memory, SCSI controller, ThinWire Ethernet port, ULTRIX Software License

$\$ 15,750$

PHIGS graphics software (covered under Cornell site license agreement)

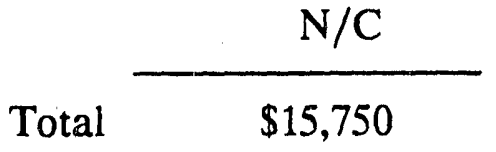

An equivalent model may be substituted at the time of final purchase if better capabilities at a similar cost become available. The proposed configuration has good interfacing capabilities with existing group hardware and software.

17 G. Stell and C.G. Joslin, "The Donnan equilibrium. A theoretical study of the effects of interionic forces" Biophys. J., 50, 855-859 (1986). 

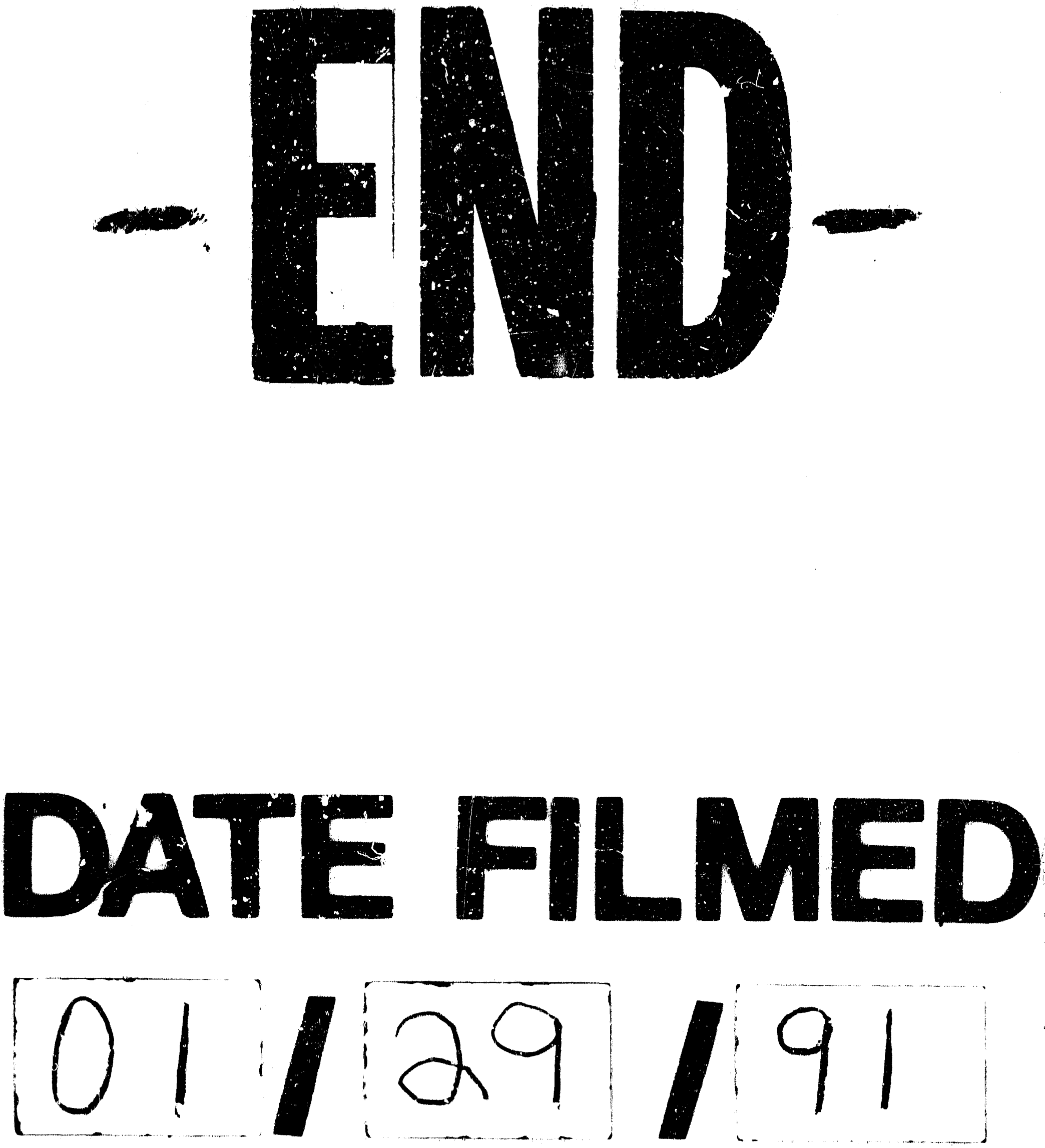
